\title{
HOMOTOPY THEORY OF RINGS AND ALGEBRAIC $K$-THEORY
}

\author{
S. M. GERSTEN
}

Communicated by P. E. Thomas, July 29, 1970

\begin{abstract}
ABSTRACr. Algebraic $K$-theory is interpreted in terms of standard homotopy notions applied to the category of rings. Representability of the functors $K^{-i}$ is discussed.
\end{abstract}

The object of this announcement is to indicate how the algebraic $K$-theory of [1] and [2] can be explained as homotopy theory in a precise sense. Some terminology of homotopy theory was used in both these articles, but the analogy turns out to be very far reaching. We work in the category $B$ of Banach rings complete in their quasinorm [2]; morphisms are bounded homomorphisms. The terminology of [2] will be assumed. From $B$ one constructs the category Hot- $B$ whose objects are those of $B$ and morphisms are homotopy classes of bounded maps (some care must be observed in defining Hot- $B$ since homotopy is per se neither transitive nor symmetric but does behave well with respect to compositions). Denote $\operatorname{Hot}-B(A, B)$ by $[A, B]$.

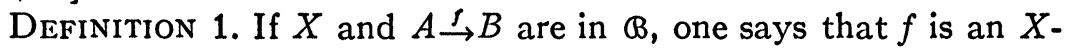
fibration if for each $n \geqq 1, E^{n} f$ induces a surjection $B\left(X, E^{n} A\right)$ $\rightarrow B\left(X, E^{n} B\right)$.

Lemma 1. For all $X$ in $B, A\{x\{\rightarrow A$ and $E A \rightarrow A$ given by " $x \rightarrow 1$ " are $X$-fibrations.

Definition 2.1 The mapping cone $C(g)$ of $g: B \rightarrow C$ is the fibre product in the diagram

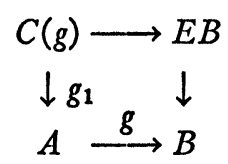

LEMMA 2. For any $X$ there is an exact sequence of pointed sets

$$
[X, C(g)] \rightarrow[X, A] \rightarrow[X, B] .
$$

AMS 1970 subject classifications. Primary 13D15, 16A54, 18F25.

Key words and phrases. Algebraic $K$-theory, homotopy in rings, Puppe sequences, representable functor, pro-rings.

1 I believe a more appropriate terminology for $E A$ and $\Omega A$ would have been the cone and suspension of $A$ respectively.

Copyright (1) 1971, American Mathematical Society 
One may iterate the construction $C(g)$ to get the diagram

$$
\cdots \rightarrow C\left(g_{n}\right) \stackrel{g_{n+1}}{\rightarrow} C\left(g_{n-1}\right) \rightarrow \cdots \rightarrow C(g) \stackrel{g_{1}}{\rightarrow} A \stackrel{g}{\rightarrow} B
$$

and the corresponding exact Puppe sequence of homotopy sets

$$
\rightarrow\left[X, C\left(g_{n}\right)\right] \rightarrow\left[X, C\left(g_{n-1}\right)\right] \rightarrow \cdots \rightarrow[X, A] \rightarrow[X, B] .
$$

Assume now that $X$ is a cogroup in $B$. That is, there is a morphism $X \rightarrow X \Perp X$ in $B$ such that $\left(1_{X} \Perp 0\right) \cdot \Delta=1_{X},\left(0 \Perp 1_{X}\right) \circ \Delta=1_{X}$ and $\left(\Delta \Perp 1_{X}\right) \circ \Delta=\left(1_{X} \Perp \Delta\right) \circ \Delta$. Then $B(X, A)$ is a group for all $A$.

Proposition 1. If $X$ is a cogroup in $B$, then the functor $\bar{X}=B(X, \cdot)$ : $B \rightarrow$ Groups is a Mayer-Vietoris Functor in the sense of [1]. In addition $[X, A]=\kappa_{1}^{\bar{x}}(A)$, where in the terminology of [1] (using the appropriate path ring $E A=x A\left\{x\{) \kappa_{1}^{\bar{X}}(A)\right.$ is defined by the exact sequence

$$
\bar{X}(E A) \rightarrow \bar{X}(A) \rightarrow \kappa_{1}^{\bar{X}}(A) \rightarrow 1 .
$$

TheOREM 1. Assume again that $X$ is a cogroup in $B$ and the diagram

$$
A \stackrel{f}{\rightarrow} B \stackrel{g}{\rightarrow} C
$$

is a short exact sequence in $B$ with $g$ an $X$-fibration. Then the exact $P$ uppe sequence above is precisely the exact $K$-theory sequence of [1]

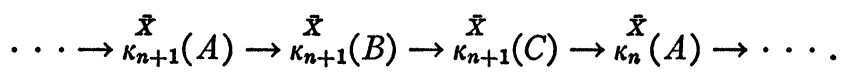

(Again, $E A$ must be suitably interpreted in [1] for nondiscrete rings.)

We may apply these notions to the functors $\mathrm{Gl}_{n}$ and Gl.

Proposition $2 . \mathrm{Gl}_{n}$ is representable by $\mathrm{gl}_{n}$ in $\mathrm{B} . \mathrm{Gl}$ is pro-representable in $₫$ by $\mathrm{gl}$. Both $\mathrm{gl}_{n}$ and $\mathrm{gl}$ are cogroups, the latter in pro-৫.

Corollary. For any $A$ in $B$ we have canonical isomorphisms $\kappa_{1}^{\mathrm{Gl}}{ }_{n}(A) \cong\left[\mathrm{gl}_{n}, A\right]$ and $K^{-1}(A)=\kappa\left({ }_{\mathrm{Gl}}^{1} A\right)=[\mathrm{gl}, A]$, where the last equation is interpreted in the pro-homotopy category. Furthermore, the exact Puppe sequence for $X=\mathrm{gl}$ in Theorem 1 is precisely the exact sequence of $[2]$ of the functors $K^{-n}$. [2].

We can also consider the representability of the functors $K^{-n}$ of

LEMMA 3. The loop ring functor $A \rightarrow \Omega A$ has an adjoint $\Sigma$ in pro-B.

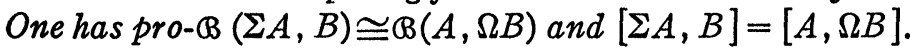

As a word of caution it should be noted that $\Sigma$ is not the suspen- 
sion functor $S$ of [2]. From Lemma 3 one deduces

Proposition 3. The functor $K^{-n}(n>0): B \rightarrow A b$ is pro-represented by $\Sigma^{n-1}$ gl.

We show in addition that $\kappa_{i}^{G}$ is pro-representable in $B$, where $G$ is a Mayer-Vietoris functor which is an algebraic group. (The definition of $\kappa_{i}^{G}$ in [1] is modified as in Proposition 1 above in the nondiscrete case.)

\section{REFERENCES}

1. S. M. Gersten, On Mayer-Vietoris functors and algebraic $K$-theory, J. Algebra (to appear).

2. M. Karoubi and O. Villamayor, Foncteurs $K^{n}$ en algèbre et en topologie, C. R. Acad. Sci. Paris 269 (1969), 416-419.

Rice University, Houston, Texas 77001 